\title{
A Compact Aberration Corrector for SEMs with Electrostatic-field formed by Annular and Circular Electrodes
}

Tadahiro Kawasaki ${ }^{1}$, Ryuji Yoshida ${ }^{1}$, Takeharu Kato ${ }^{1}$, Tsunenori Nomaguchi ${ }^{2}$, Shunichi Motomura ${ }^{2}$, Kenichi Nishinaka ${ }^{2}$, Toshihide Agemura ${ }^{2}$, and Takashi Ikuta ${ }^{3}$

1. Nanostructures Research Laboratory, Japan Fine Ceramics Center, Nagoya, Japan

2. Hitachi High-Technologies, Hitachinaka, Japan,

${ }^{3}$. Faculty of Engineering, Osaka Electro-Communication University, Neyagawa, Japan

The spherical aberration (Cs) correction technique has almost been established especially for (scanning) transmission electron microscopes ((S)TEMs) to improve spatial resolution. On the other hand, for the scanning electron microscopes (SEMs), this technique had been developed but not utilized widely for applications though SEMs are also requested to be higher spatial resolution. This is because the conventional Cs correctors require complex control of several optical components with high accuracy and stability, so that the size and the cost of the corrector are too large to install into SEMs. In order to solve these problems, the authors had proposed a simple and compact Cs-corrector with axially-symmetric electrostatic-field formed between annular and circular electrodes [1,2], as schematically shown in Fig. 1(a), so called "ACE corrector" (the Cs-corrector using Annular and $\underline{\text { Circular }}$ Electrodes). We have been developing the ACE corrector system for installing the conventional SEM; SU5000 (Hitachi High-Technologies Corp.) and have been optimizing experimental parameters to obtain higher performances, such as sizes of electrodes, alignment precision, and so on.

One of the problems on the ACE corrector is diffraction effect by the annular electrode. Figs. 1(b) and (c) show simulation results of probe intensity distributions calculated in uncorrected and Cs corrected conditions, respectively. The ACE corrector can generate negative Cs by electrostatic field around the annular electrode. Electron beam trajectories passing through the annular slit are spread, indicating that annular electrode acts as a concave lens approximately. The annular electrode has another advantage to reduce chromatic aberration effect by elongating the focal depth as a quasi-Bessel beam. However, the annularly-restricted electron beam provides not only a sharp center peak but also widely distributed intensities around the main peak, as shown in Fig. 1(c). The effect of the sub-peaks appears as undesired fringes at object edges and background increment in SEM images, as shown in Fig. 2(a). These artifacts are caused by lack of middle spatial frequency components due to the annular slit (Fig. 2(b)). They can be compensated by applying a Fourier filter to the images, determined as the auto-correlation function of the annular pupil (Fig. 2(c)). After the filtering process, incorrect fringes and foggy background around the objects clearly disappear, as shown in Fig. 2(d). This indicates that the proposed image processing is effective for the ACE corrector. 


\section{References}

[1] T. Kawasaki, et al., Suf. Int. Anal, 48 (2015) 1160

[2] T. Kawasaki, et al., PCT/JP2016/053691

\section{Acknowledgement}

This development was supported by SENTAN, JST.
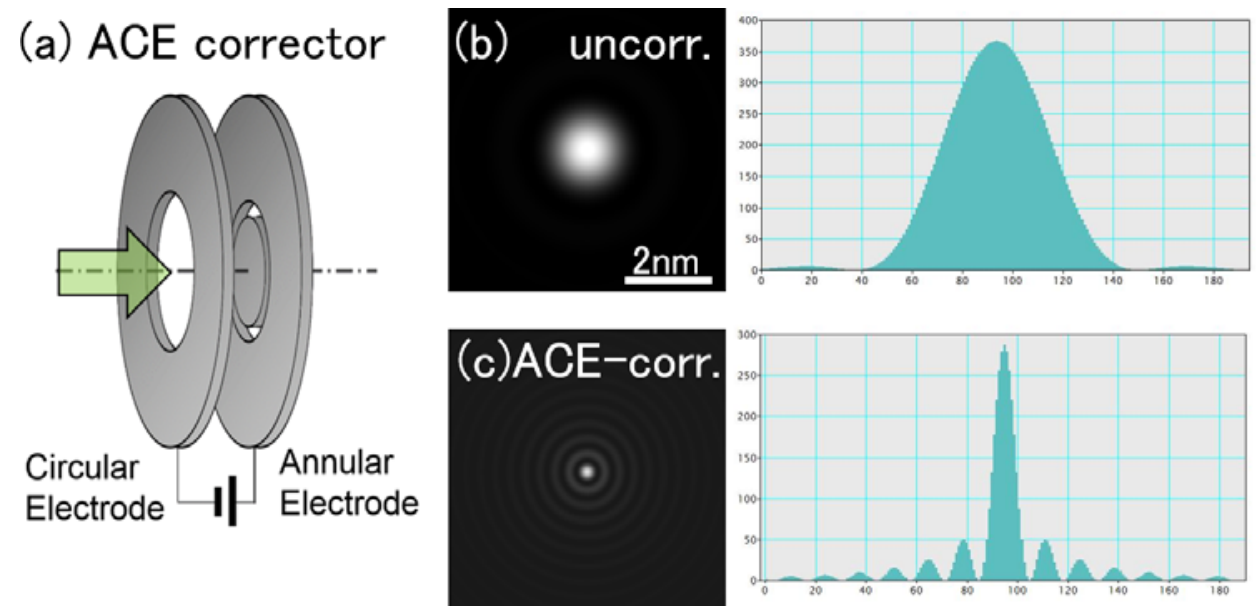

Figure 1. (a)Schematic illustrations of the ACE corrector. (b) Simulated probe intensity distributions at (b) uncorrected and (c) corrected conditions. $\mathrm{V}_{\text {acc }}=30 \mathrm{kV}$.
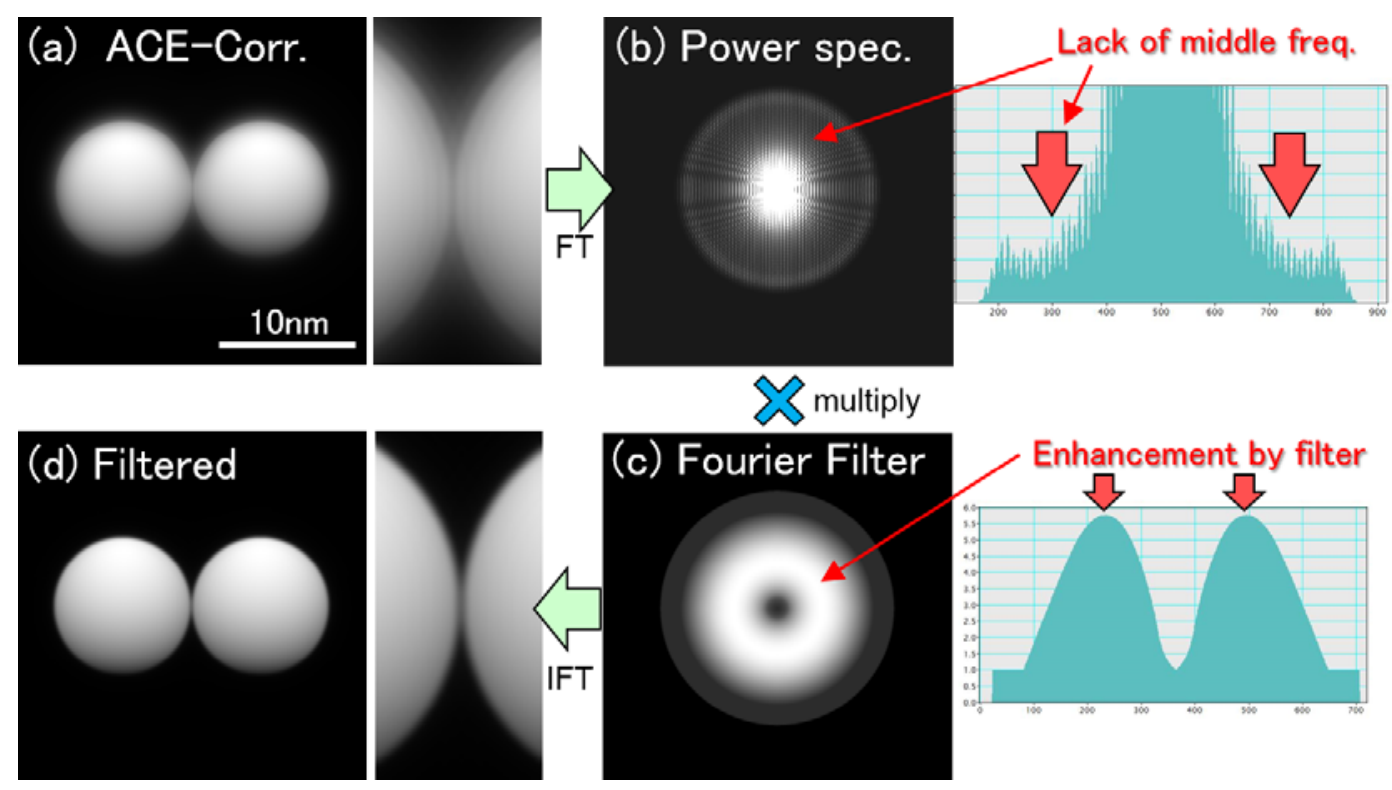

Figure 2. (a) Simulated SEM image of nanoparticles having $10 \mathrm{~nm}$ in diameter with Cs correction. (b) Power spectrum of (a) and its line profile. (c) Middle-range enhancement Fourier filter dedicated for (b). (d) Filtered SEM image by using (c), in which no edge fringes and foggy background are visible. $\mathrm{V}_{\text {acc }}=30 \mathrm{kV}$. 